\title{
Study of Dye Absorption in Carbon Nanotube-Titanium Dioxide Heterostructures
}

\author{
Marianna Barberio ${ }^{*}$, Alessandra Imbrogno, Davide Remo Grosso, Assunta Bonanno and Fang Xu \\ DiBEST \& DiMEG Department, University of Calabria, INFN-CS 87036, Italy
}

\begin{abstract}
In this work, the authors present a study of dye absorption in $\mathrm{TiO}_{2}$ doped with $\mathrm{CNTs}$ (carbon nanotubes). Absorption decreases exponentially with the increase of CNTs in the film, while morphological characterization, conducted by SEM (scanning electron microscope) and TEM (transmission electron microscope) microscopes, suggests that this behavior is strongly related to morphological structure of grown films. For CNTs amounts greater than $1 \%$, the authors observe the formation of CNTs clusters randomly distribute on $\mathrm{TiO}_{2}$ bulk, which strongly reduces the film porosity quenching the dye absorption. Comparison with optical properties of $\mathrm{CNT} / \mathrm{TiO}_{2}$ filmstudied in the previous work, suggest that the best level of doping is with $0.5 \%$ of CNTs. FTIR (Fourier transform infrared spectroscopy) measurements conducted on a series of pristine and doped samples clearly indicate the absence of change in allotropic species of $\mathrm{TiO}_{2}$, while AFM (atomic force microscope) analysis indicates that the sample roughness strongly changes with doping, preventing the dye adsorption. Finally, measurements of cell efficiency indicate an increase of 5\% in cells with $0.5 \%$ of CNT doping and a decrease for greater values.
\end{abstract}

Key words: Graetzel cells, dye adsorption, titanium dioxide, carbon nanotube doping.

\section{Introduction}

Although solid-state junction devices with high efficiency dominate the commercial market, these photovoltaic technologies still receives constraints in market development due to both of expensive materials and complex manufacturing process. Consequently, the emerging photovoltaic technologies such as organic and inorganic cells, quantum dots and DSSCs (dye sensitized solar cells), have attracted extensive attention because of their promising inexpensive technique based on the solution-processed materials. However, these new devices seem to be associated with unsatisfactory efficiency (with value that ranges between $7 \%$ and $20 \%$ on laboratory prototypes) [1-3].

DSSCs mainly consist of a dye-sensitized $\mathrm{TiO}_{2}$ film deposited onto a transparent conducting substrate as the photoanode (working electrode, WE), a redox active electrolyte and a Pt cathode (counter electrode, CE). The low efficiency of DSSCs is linked to

\footnotetext{
*Corresponding author: Marianna Barberio, Ph.D., research field: nanotechnology. E-mail: marianna.barberio@fis.unical.it.
}

different factors as dye deterioration, electron/hole recombination in $\mathrm{TiO}_{2}$-dye substrate, contact resistance between $\mathrm{CE}$ and electrolyte. Research on DSSC focuses on two different DSSC: (1) Semiliquid DSSCs where the photosensitive element is an organic dye (i.e. N719) (2) Semisolid DSSCs where the photosensitive element is a thin film of semimetal quantum dots ( $\mathrm{ZnS}, \mathrm{CdS}, \mathrm{PbS})$. In the first field, many efforts have been dedicated to the study of new micro and nanostructured materials for both photo anode and counter electrodes. The correct choice of materials at photoanode is crucial because the phenomena of generation of photoelectrons and their passage to electric circuit are the most delicate physical steps in a DSSC and little change in their mechanism can strongly change (enhancing or decreasing) cell efficiency. The best material used in a classical DSSC is nanoporous $\mathrm{TiO}_{2}$ thin films filled by the liquid dye $[2,3] . \mathrm{TiO}_{2}$, usually used in both mineral phases (anatase and rutile), supplies several advantages due to its high chemical stability, excellent functionality, nontoxicity, optical proprieties (both are transparent in 
the visible and absorbed in the near ultraviolet region [3]) and relatively low cost. Great efforts have been made in the last years to enhance the properties of $\mathrm{TiO}_{2}$ by the doping with carbon-based materials. Carbon is generally considered as one of the best candidates for dopants because theoretical and experimental studies [4, 5-9] have demonstrated the possibility to reduce drastically the band gap of $\mathrm{TiO}_{2}$ and to improve the adsorption in the visible range. In our previous works [10] we obtained great enhancement in the optical properties of carbon-doped $\mathrm{TiO}_{2}$ substituting the simple doping with carbon atoms with CNTs (carbon nanotubes). We obtain good results in photoluminescence quenching and optical bands reduction with very low weight percent ( $\mathrm{wt} \%)$ of CNT. In our hybrid $\mathrm{CNT} / \mathrm{TiO}_{2}$ films the luminescence emission is strongly quenched with respect to pure $\mathrm{TiO}_{2}$ with a sensible reduction in the visible region [9-13], while the optical band is reduced of $0.6 \mathrm{eV}$ for microstructured and $0.1 \mathrm{eV}$ for nanostructured films [13].

However, the insertion of photoanode with CNT amount ranging from $1 \%$ to $3 \%$ in DSSC cells causes a fall in cell efficiency of about $13 \%$ respect to a classical cell [14]. This work explains this unexpected behavior of new materials in terms of decrease of dye absorption with doping, suggesting the right amount of CNT doping which ensure enhancement in optical properties without sensible loss in dye absorption.

\section{Experiments}

The titanium dioxide used in this work is a commercial powder (purchased from Advanced Materials) of rutile mainly composed of spherical grains with dimensions of about $200 \mathrm{~nm}$. The carbon nanotubes used to growth the nano-hybrid materials are commercial powders of bundles of multiwalled carbon nanotubes (purchased by Nano-Lab co. USA). The MWCNT (multiwalled carbon nanotubes) buckypaper has a nominal purity greater than $95 \%$ and at SEM analysis, shows a disordered structure composed by a network of tube bundles with length of the order of $10 \mu \mathrm{m}$ and a diameter of about $20-30 \mathrm{~nm}$.

The film of Titanium dioxide was prepared as the standard indication for $\mathrm{TiO}_{2}$ film in DSSCs [15]. $0.2 \mathrm{~g}$ of rutile was first dissolved in $0.4 \mathrm{~mL}$ of acetic acid and, then, a uniform paste was prepared adding 15.52 $\mu \mathrm{L}$ of Acetilacetone, $3.24 \mu \mathrm{L}$ of Triton X-100 and 0.4 $\mathrm{mL}$ of PEG2000 aqueous solution.

The CNT doped samples were obtained mixing CNT with rutile powder, before the film preparation process. We prepare a series of CNT-doped films with weight percentage of CNT ranging from $0.1 \%$ to $5 \%$. The obtained paste was then spin coated on a glass substrate and heated in air at $400{ }^{\circ} \mathrm{C}$ for $30 \mathrm{~min}$.

An aqueous solution $(80 \mu \mathrm{L}) 2.62 \mathrm{mM}$ of N719 (Cis-Bis(isotiocianato)Bis(2,2'-bipiridil-4,4'-dicarbos silato) rutenio (II)) were uniformly deposed on each prepared films and heated at about $50{ }^{\circ} \mathrm{C}$ for $1 \mathrm{~min}$.

For obtaining N719 desorption from rutile, each prepared film was inserted in $40 \mathrm{~mL}$ of a solution 0.1 $\mathrm{M}$ of $\mathrm{NaOH}$ in $\mathrm{EtOH} / \mathrm{H}_{2} \mathrm{O}$. After the complete desorption of $\mathrm{N} 719$ the absorbance spectra of colloidal solution was taken (as the process described in [16]) to estimate the concentration of desorbed molecules. Measurements of optical absorption were obtained irradiating the solution by a white lamp (Energetiq LDLS, laser driven light source), and taken the transmitted spectra by a Triax 320 (Horiba-Jobyn-Yvon) spectrometer working in the 300-800 nm range. The extinction cross section and optical absorption were then evaluated using the standard Eqs. (1) and (2):

$$
\begin{gathered}
\in(\lambda)=-\log \frac{I_{t}(\lambda)}{I_{0}(\lambda)} \\
a(\lambda)=1-\frac{I_{t}(\lambda)}{I_{0}(\lambda)}
\end{gathered}
$$

where, $I_{t}$ and $I_{0}$ are, respectively, the transmitted and source intensity at each wavelength.

The particle concentration was evaluated following the Lambert and Beer's Law:

$$
a=c \alpha l
$$


where, $c$ is the particle concentration, $a$ is the molar extinction cross section and $l$ is the optical path length in our experimental setup.

Morphological information was taken by SEM (scanning electron microscope), AFM (atomic force microscope) and TEM (transmission electron microscope). SEM images were taken under a STEREOSCAN SEM microscope working with energy of $20 \mathrm{keV}$. AFM images were obtained by the ICON AFM microscope from Bruker working in tapping mode. Each image was taken with a resolution of $512 \times 512$ pixels and a frequency of $1 \mathrm{~Hz}$. The images were, then, elaborated by the nanoscope software (1.40 version from Bruker) to obtain morphological information and surface roughness.

FTIR (Fourier transform infrared spectroscopy) measurements were conducted by a Perkin Elmer Spectrum 100, equipped with a universal ATR (attenuated total reflectance) accessory.

Finally, the produced materials have been tested in DSSC cells realized using as counter electrode an amorphous carbon films deposed on a FTO (Fluorine-thin oxide) glass, as electrolyte solution the couple $\mathrm{I}^{3-} / \mathrm{I}^{-}$, and as photoanode pure rutile, CNT $(0.5 \%) /$ rutile, or CNT $(1 \%) /$ rutile composites, prepared as discussedabove and spin-coated on a FTO

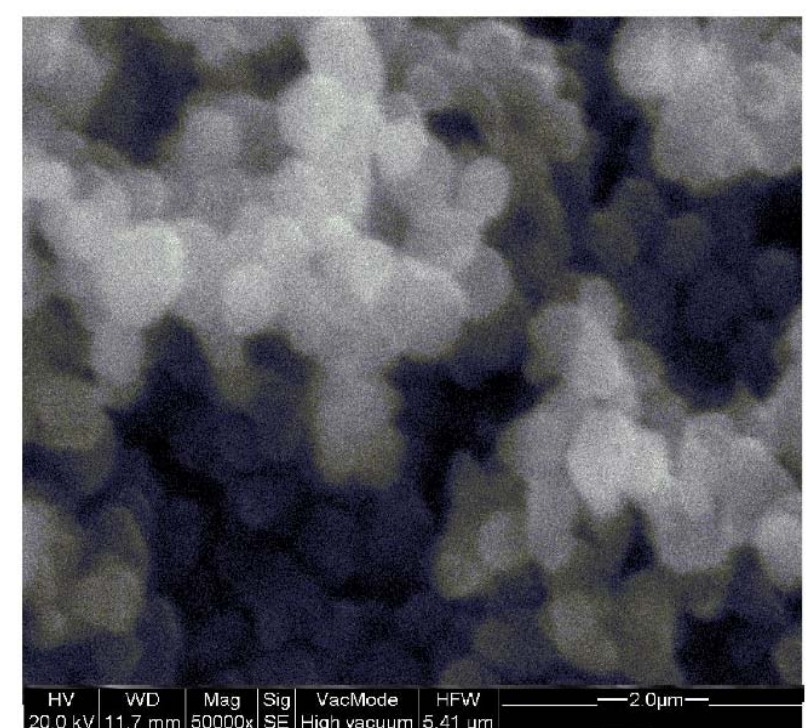

(a) glass substrate (film thickness of $1.5 \mu \mathrm{m}$ ).

\section{Results and Discussion}

SEM (Fig. 1) and TEM (Fig. 2) images of our prepared $\mathrm{CNT} / \mathrm{TiO}_{2}$ hybrid films clearly indicate that the sample morphology change strongly with the increases of CNT amount. For low concentration of CNT $(0.1 \%-1 \%)$ the films appear as a bulk of rutile grains with randomly insertion of carbon nanotubes, the tubes fill the interstices between grains without cover the grains or changing the film structure. In this case, we can associate the mixing to doping process of rutile bulk. For higher CNT amount (1\%-5\%) the film structure changes, at SEM images the film appears as a bulk of rutile grains with insertions of large clusters of CNT. Finally, for amount of CNT $>5 \%$ we observe the formation of a grainy structure composed by well separated cluster of CNT and rutile, the film is inhomogeneous and cannot be identified as a single material or compound.

Moreover, FTIR spectra taken from our heterostructures (Fig. 3) indicate, in all samples, the presence of the only rutile phase (band at $471 \mathrm{~cm}^{-1}$ ) $[17,18]$, confirming the absence of chemical interactions between carbon nanotubes and titanium dioxide.

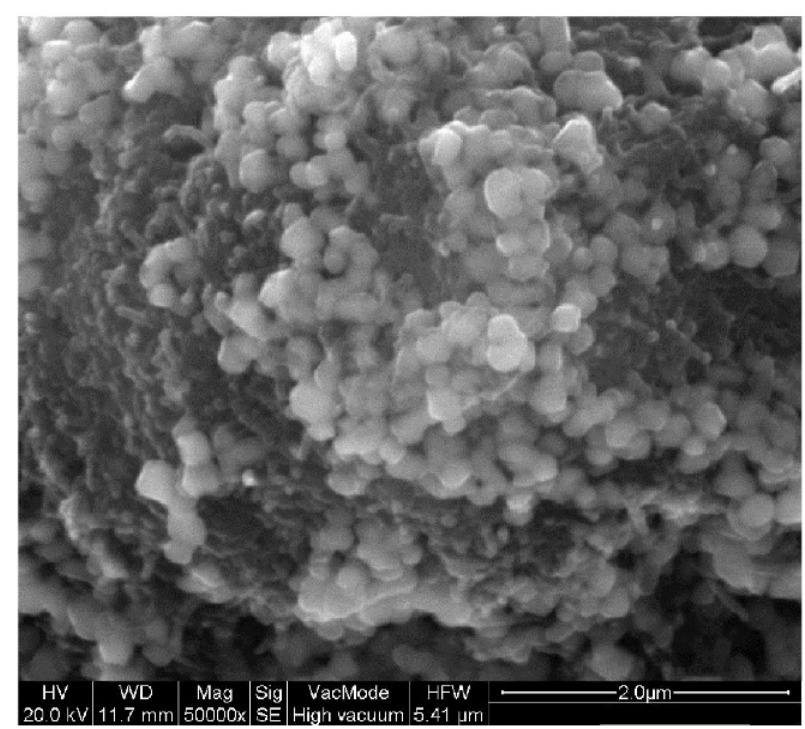

(b)

Fig. 1 SEM images of $\mathrm{CNT} / \mathrm{TiO}_{2}$ hybrid films with different wt $\%$ of CNT: $0.5 \%$ (a), and $5 \%$ (b). 


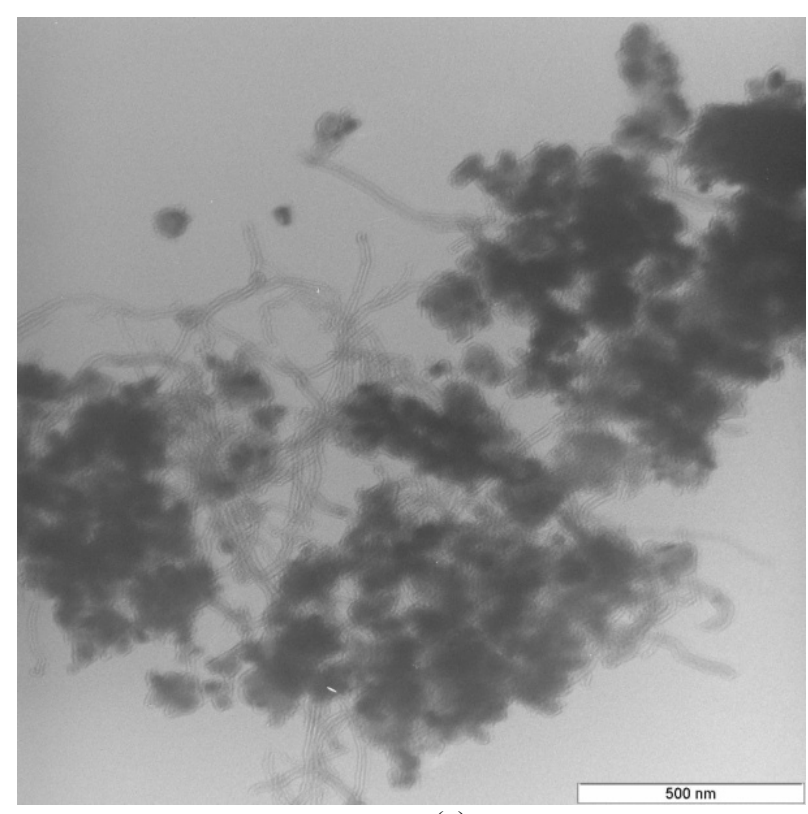

(a)

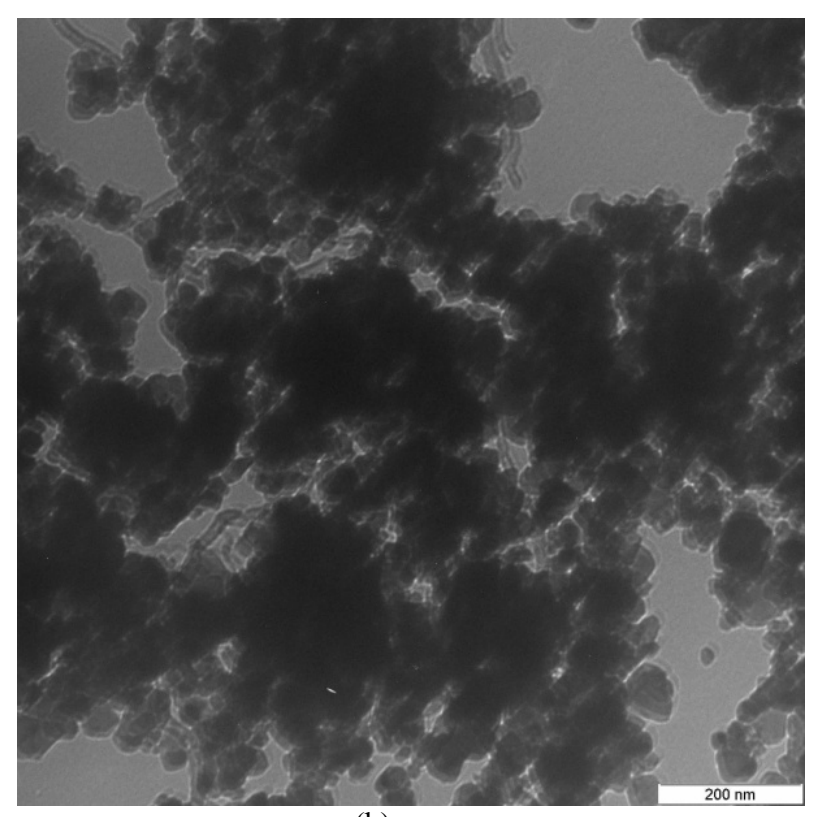

(b)

Fig. 2 TEM images of $\mathrm{CNT} / \mathrm{TiO}_{2}$ hybrid films with different wt $\%$ of $\mathrm{CNT}$ : $0.5 \%$ (a) and $5 \%$ (b).

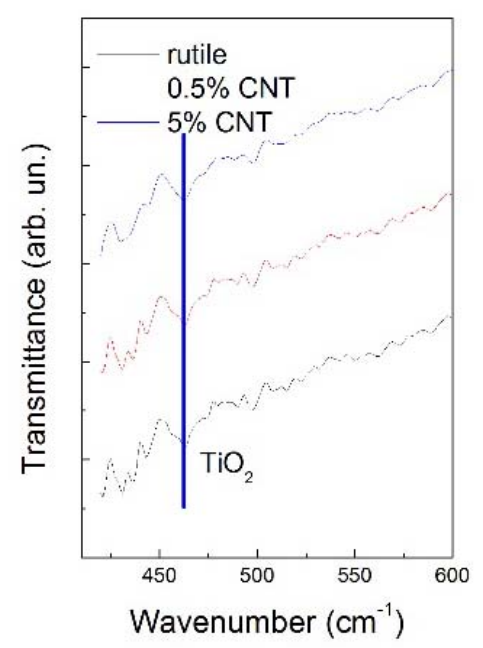

Fig. 3 FTIR spectra taken on pure rutile, CNT $(0.5 \%) /$ rutile, and CNT (5\%)/rutile.

The dye absorption of rutile film changes strongly with the CNT amount. The absorption extinction coefficient in Fig. 4a clearly indicates that the spectrum collected for pure rutile is very different from these collected from film with $5 \%$ or $1 \%$ of CNT. The intensity of extinction decreases of about $70 \%$ indicating the fall of N719 absorption in the inhomogeneous and grainy films. The Gaussian analysis of extinction cross section of pure rutile films (Fig. 4b) reveals a structure with two main plasmonic oscillation at 518 and $580 \mathrm{~nm}$ indicating the

absorption of particles with diameter of about 30-50 $\mathrm{nm}$ (estimated by the electrodynamic Mie-Gans theory). The spectra collected for absorbance in doped film (Fig. 4c) shows a red shift of plasmonic peaks at 540 and $600 \mathrm{~nm}$ clearly indicating the absorption of greater particles with diameter of 40-70 nm. We suggest that this behavior is caused by the absence of small pores on films with high amount of CNT. These samples are inhomogeneous and the presence of big cluster of CNT reduces the absorption properties of rutile porous structures.

AFM morphological analysis (Fig. 5) conducted on pure $\mathrm{TiO}_{2}$ (Fig. 5a) and on $\mathrm{TiO}_{2}$ doped with $0.5 \%$ of CNT (Fig. 5b) indicates that samples roughness increases of about $25 \%$ (passing from $41 \mathrm{~nm}$ to $51 \mathrm{~nm}$ ). While in samples with greater amounts of CNTs, the regions with CNTs are completely separated from those with rutile, as observed in all morphological characterizations, and the sample roughness is characteristic of pure rutile $(41 \mathrm{~nm})$ in $\mathrm{TiO}_{2}$ areas while istypical of pure CNT $(120 \mathrm{~nm})$ for tube clusters. The absorption of dye in non-uniform hybrids is, then, strongly different in $\mathrm{TiO}_{2}$ and $\mathrm{CNT}$ areas. In particular, the presence of large CNT areas with high roughness reduces the dye absorption and, then, the cell efficiency. 


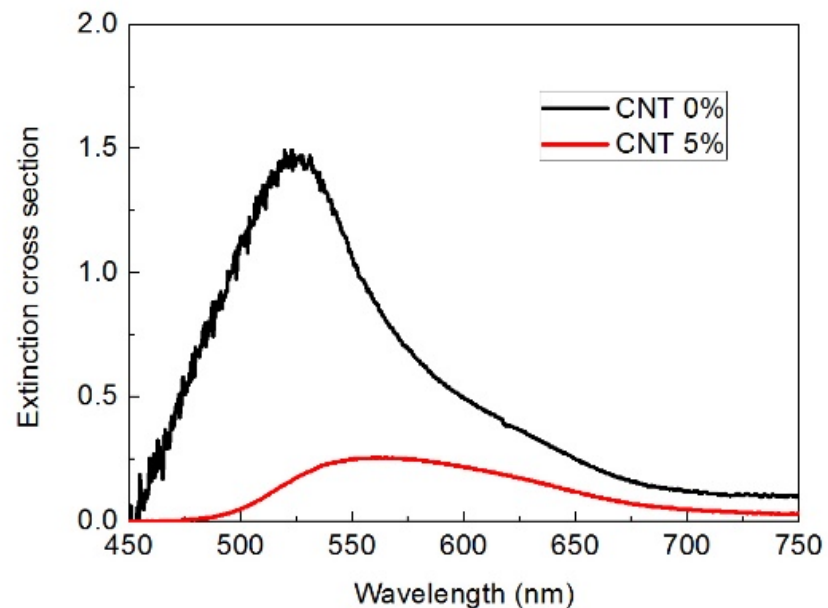

(a)

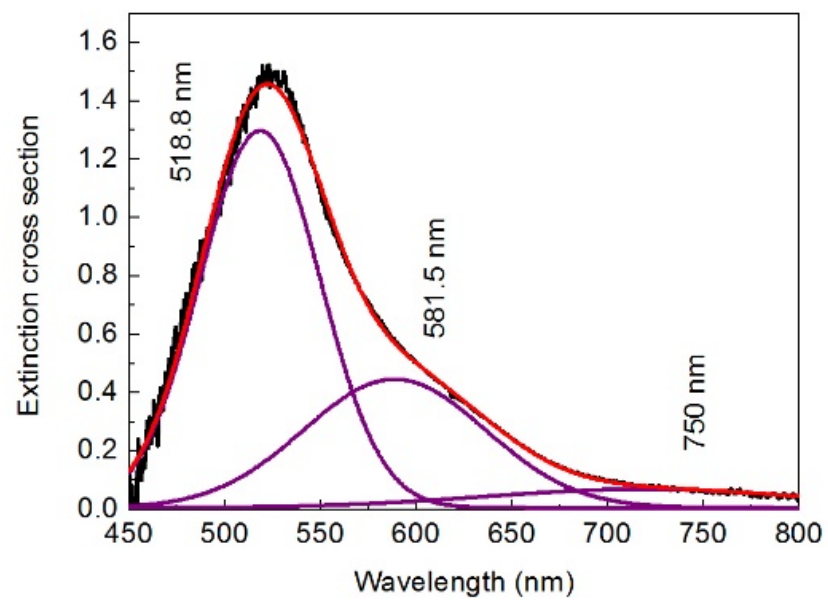

(b)

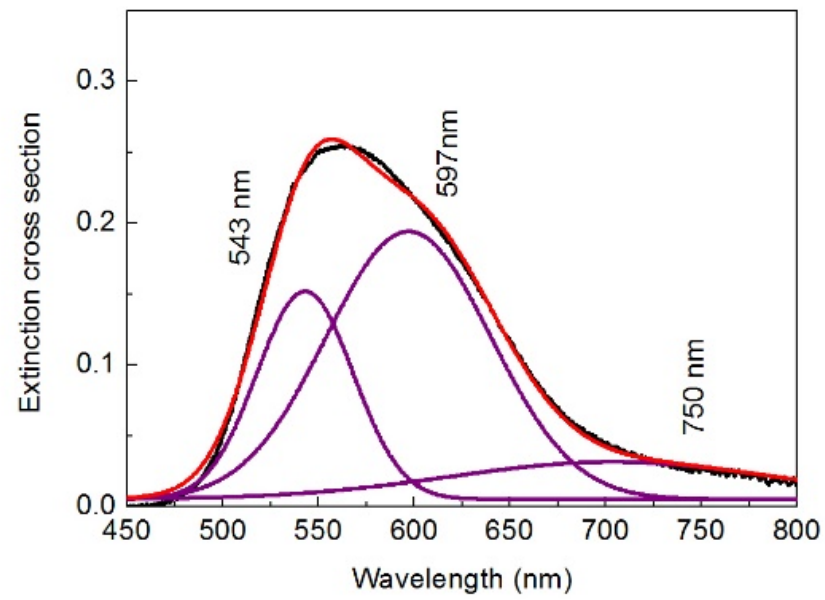

(c)

Fig. 4 Extinction cross section for desorbed dye from hybrid film with 1\% and 5\% of CNT (a) and their Gaussian analysis (b and c for $0 \%$ and $5 \%$ respectively). 


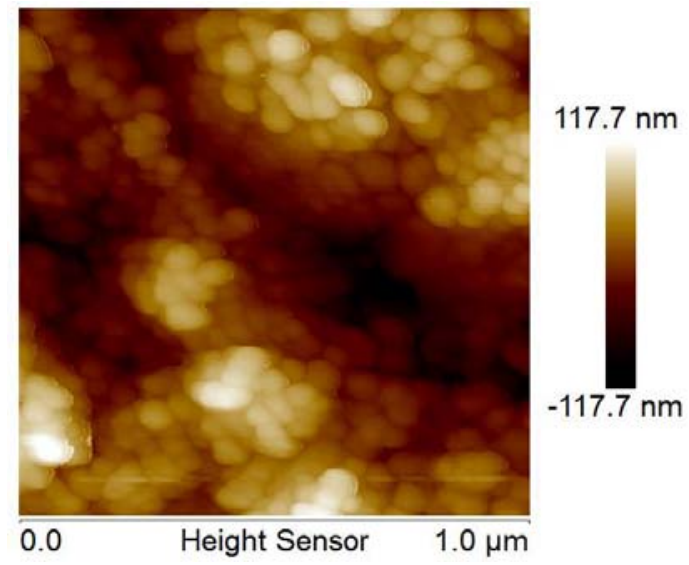

(a)

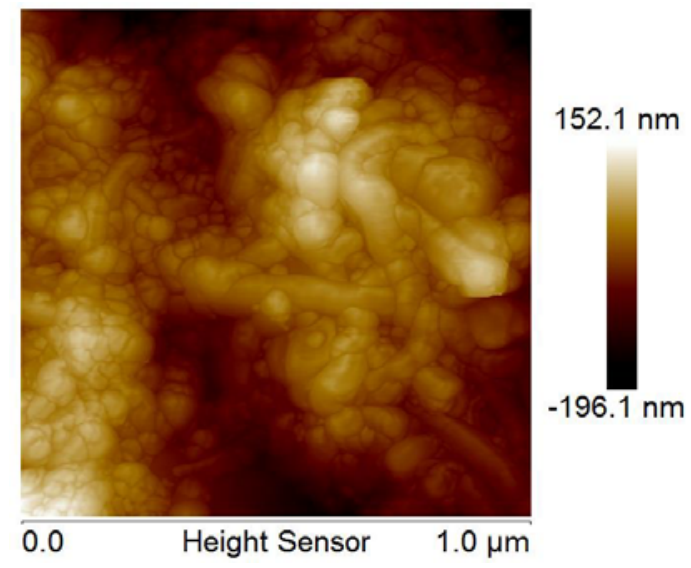

(b)

Fig. 5 AFM images of pure $\mathrm{TiO}_{2}$ (a) and $0.5 \% \mathrm{CNT} / \mathrm{TiO}_{2}$ hybrid film (b).

In the other hands, the total amount of adsorbed particles (Fig. 6) decreases exponentially with the CNT amount. This clearly indicates that the doping with carbon nanotubes strongly reduces the ability of $\mathrm{TiO}_{2}$ to absorbed dye molecules. This phenomenon can results in a decreases of dye solar cells efficiency caused by the reduction of dye amount in cells.

As discussed in Introduction section, scientific results of our previous works in [13], indicate in the mix of anatase or rutile with carbon nanotube the best way to enhance the optical absorption of titanium dioxide in visible region and to quench the emission of photoluminescence. Both these properties are useful to enhance cell efficiency and increase their effectiveness with the amount of CNT. In [13] we observed a variation of $\Delta \phi=0.1 \mathrm{eV}$ in optical gap for nanometric rutile or anatase at a concentration of CNTs of about $1.5 \mathrm{wt} \%$ and a corresponding quenching of luminescence intensity of about $50 \%$. Here, we can observe that for the same CNT amount the dye absorption in photoanode decreases of about $60 \%$, so that the benefits introduced by the CNT in the optical properties of titanium dioxide are completely balanced by loss in dye adsorption. The best doping will be reached for a CNT wt.\% equal to $0.5 \%$ the decreases in dye absorption is about of $20 \%$ while the quenching of photoluminescence intensity and the decreases of optical band are $20 \%$ and $1.5 \%$, for CNT amount greater than $0.5 \%$ the dye absorption fall to value that can strongly reduce the cell efficiency.

Finally, measurements of cell efficiency on DSSCs (Fig. 7) confirm our results, indicating an increase respect to classical cells of about $5 \%$ for photoanode with $0.5 \%$ of CNT and a decrease of 10 for $1 \%$ of tubes.

\section{Conclusions}

SEM (Fig. 1) and TEM (Fig. 2) images of our prepared $\mathrm{CNT} / \mathrm{TiO}_{2}$ hybrid films clearly indicate that the sample morphology change strongly with the increases of CNT amount. For low concentration of CNT $(0.1 \%-1 \%)$ the films appear as a bulk of rutile grains with randomly insertion of CNTs, the tubes fill the interstices between grains without cover the grains

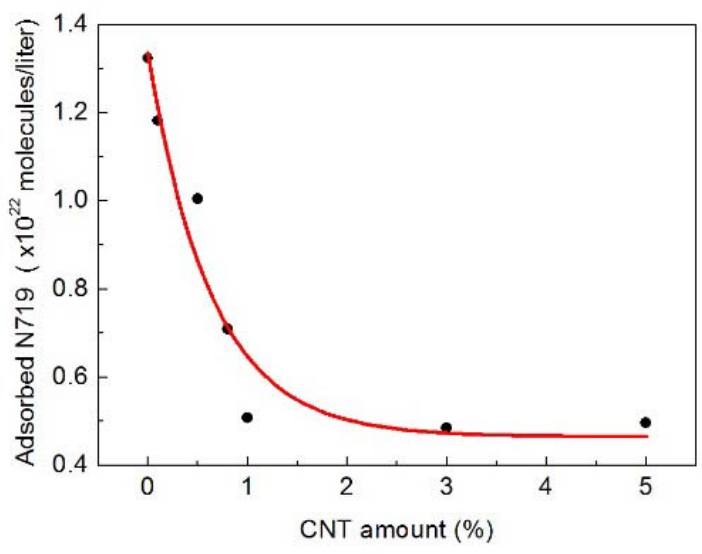

Fig. 6 Desorbed dye as function of CNT wt \%. 


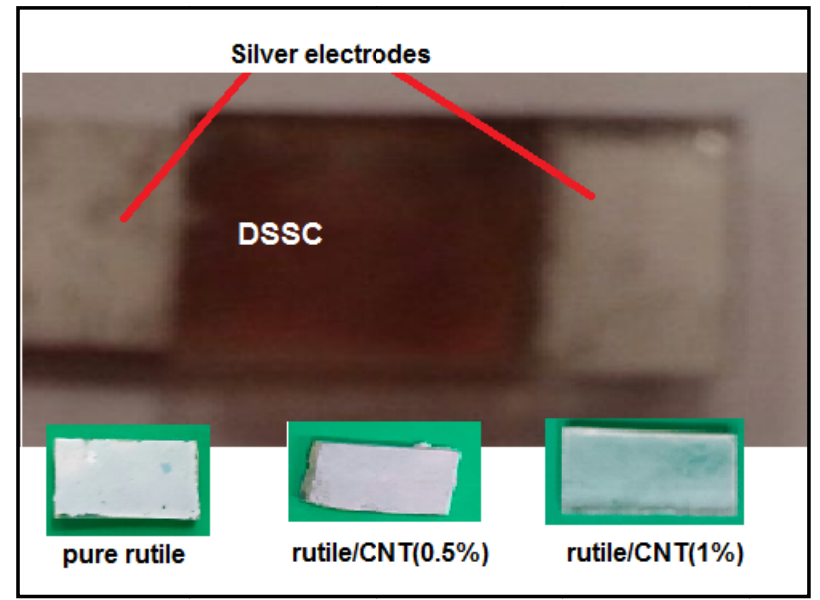

Fig. 7 DSSC cells realized with a photoanode of pure rutile or CNT $(0.5 \%) /$ rutile or CNT $(1 \%) /$ rutile.

or changing the film structure. In this case, the authors associate the mixing to doping process of rutile bulk. For higher CNT amount (1\%-5\%) the film structure changes, at SEM images the film appears as a bulk of rutile grains with insertions of large clusters of CNT. Finally, for amount of CNT $>5 \%$ we observe the formation of a grainy structure composed by well separated cluster of CNT and rutile, the film is inhomogeneous and cannot be identified as a single material or compound.

Moreover, FTIR spectra taken from our heterostructures (Fig. 3) indicate, in all samples, the presence of the only rutile phase (band at $471 \mathrm{~cm}^{-1}[17$, 18]), confirming the absence of chemical interactions between carbon nanotubes and titanium dioxide.

The dye absorption of rutile film changes strongly with the CNT amount. The absorption extinction coefficient in Fig. 4a clearly indicates that the spectrum collected for pure rutile is very different from these collected from film with $5 \%$ or $1 \%$ of CNT. The intensity of extinction decreases of about $70 \%$ indicating the fall of N719 absorption in the inhomogeneous and grainy films. The Gaussian analysis of extinction cross section of pure rutile films (Fig. 4b) reveals a structure with two main plasmonic oscillation at 518 and $580 \mathrm{~nm}$ indicating the absorption of particles with diameter of about 30-50 $\mathrm{nm}$ (estimated by the electrodynamic Mie-Gans theory). The spectra collected for absorbance in doped film (Fig. 4c) show a red shift of plasmonic peaks at 540 and $600 \mathrm{~nm}$ clearly indicating the absorption of greater particles with diameter of 40-70 nm. We suggest that this behavior is caused by the absence of small pores on films with high amount of CNT. These samples are inhomogeneous and the presence of big cluster of CNT reduces the absorption properties of rutile porous structures.

AFM morphological analysis (Fig. 5) conducted on pure $\mathrm{TiO}_{2}$ (Fig. 5a) and on $\mathrm{TiO}_{2}$ doped with $0.5 \%$ of CNT (Fig. 5b) indicates that samples roughness increases of about $25 \%$ (passing from $41 \mathrm{~nm}$ to 51 $\mathrm{nm})$. While in samples with greater amounts of CNTs the regions with carbon nanotubes are completely separated from those with rutile, as observed in all morphological characterizations, and the sample roughness is characteristic of pure rutile $(41 \mathrm{~nm})$ in $\mathrm{TiO}_{2}$ areas while istypical of pure CNT $(120 \mathrm{~nm})$ for tube clusters. The absorption of dye in non-uniform hybrids is, then, strongly different in $\mathrm{TiO}_{2}$ and CNT areas. In particular, the presence of large CNT areas with high roughness reduces the dye absorption and, then, the cell efficiency.

In the other hands, the total amount of adsorbed particles (Fig. 6) decreases exponentially with the CNT amount. This clearly indicates that the doping with carbon nanotubes strongly reduces the ability of $\mathrm{TiO}_{2}$ to absorbed dye molecules. This phenomenon can results in a decreases of dye solar cells efficiency caused by the reduction of dye amount in cells.

As discussed in introduction section, scientific results of our previous works in [13], indicate in the mix of anatase or rutile with carbon nanotube the best way to enhance the optical absorption of titanium dioxide in visible region and to quench theemission of Photoluminescence. Both these properties are useful to enhance cell efficiency and increase their effectiveness with the amount of CNT. In [13] we observed a variation of $\Delta \phi=0.1 \mathrm{eV}$ in optical gap for nanometric rutile or anatase at a concentration of 
CNTs of about 1.5 wt.\% and a corresponding quenching of luminescence intensity of about $50 \%$. Here, we can observe that for the same CNT amount the dye absorption in photoanode decreases of about $60 \%$, so that the benefits introduced by the CNT in the optical properties of titanium dioxide are completely balanced by loss in dye adsorption. The best doping will be reached for a CNT wt.\% equal to $0.5 \%$ the decreases in dye absorption is about of $20 \%$ while the quenching of photoluminescence intensity and the decreases of optical band are $20 \%$ and $1.5 \%$, for CNT amount greater than $0.5 \%$ the dye absorption fall to value that can strongly reduce the cell efficiency.

Finally, measurements of cell efficiency on DSSCs (Fig. 7) confirm our results, indicating an increase respect to classical cells of about $5 \%$ for photoanode with $0.5 \%$ of CNT and a decrease of 10 for $1 \%$ of tubes.

\section{References}

[1] Hemissi, M., and Amardjia-Adnani, H. 2007. "Optical and Structural Properties of Titanium Oxide Thin Films Prepared by Sol-Gel Method." Digest Journal of Nanomaterials and Biostructures 2 (4): 299-305.

[2] Sabataityté, J., Oja, I., Lenzman, F., Volobujeva, O., and Krunks, M. 2006. "Characterization of Nanoporous $\mathrm{TiO}_{2}$ Films Prepared by Sol-gel Method.” Comptes Rendus Chimie 9 (5-6): 708-12.

[3] Zallem, R., and Moret, M. P. 2006. "The Optical Absorption Edge of Brookite $\mathrm{TiO}_{2}$." Solid State Communications 137: 154-7.

[4] Kaneko, M., and Ohkura. 2002. Photocatalysis. Tokyo: Springer.

[5] Kamisaka, H., Adachi, T., and Yamashitaa, K. 2005. "Theoretical Study of the Structure and Optical Properties of Carbon-doped Rutile and Anatase Titanium Oxides." Journal of Chemical Physics 123: 084704-13.

[6] Choi, W., Termin, A., and Hoffmann, M. R. 1994. "The Role of Metal Ion Dopants in Quantum-Sized $\mathrm{TiO}_{2}$ : Correlation between Photoreactivity and Charge Carrier Recombination Dynamics." Journal of Physical Chemistry 98: 13669-79.

[7] Wang, H., and Lewis, J. P. 2006. "Second-generation Photocatalytic Materials: Anion-doped $\mathrm{TiO}_{2}$." Journal of
Physics: Condensed Matter 18: 421-34.

[8] Pascual, J., Camassel, J., and Mathieu, H. 1978. "Fine Structure in the Intrinsic Absorption Edge of $\mathrm{TiO}_{2}$." Physical Review B 18: 5606-14.

[9] Barberio, M., Barone, P., Imbrogno, A., Xu, F., Pingitore, V., and Bonanno, A. 2012. "Optical and Structural Properties of Carbon Nanotube-Rutile Heterostructures." Journal of Chemistry and Chemical Engineering 6 (10): 942-50.

[10] Barberio, M., Barone, P., Bonanno, A., and Oliva, A. 2012. "Synthesis and Characterization of Carbon Nanotubes Wrapped on Anatase Microparticles.” Particle and Particle Systems Characterization 28: 64-70.

[11] Barberio, M., Barone, P., Pingitore, V., and Bonanno, A. 2012. "Optical Properties of $\mathrm{TiO}_{2}$ Anatase-Carbon Nanotubes Composites Studied by Cathodoluminescence Spectroscopy." Superlattice and Microstructure 51: 177-83.

[12] Barberio, M., Barone, P., Imbrogno, A., Ruffolo, A. S., La Russa, M., Arcuri, N., and Xu, F. 2014."Study of Band Gap of Carbon Nanotube-Titanium Dioxide Heterostructures." Journal of Chemistry and Chemical Engineering 8 (1): 36-41.

[13] Condrò, L. 2014. “Chemical Engineering.” Master Thesis, University of Calabria.

[14] Ito, S, Murakami, T.N., Comte P, Liska P, Graetzel C, Nazeeruddin, M. K., and Graetzel, M. 2008. "Fabrication of Thin Film Dye Sensitized Solar Cells with Solar to Electric Power Conversion Efficiency over 10\%." Thin Solid Films 516: 4613-19.

[15] Barberio, M., Barone, P., Xu, F., and Bonanno, A. 2013. "Silver Nanoparticles Synthesized by Laser Ablation in Acetone: Influence of Ablation Time and Their Reactivity with Oxygen in the Air." Journal of Chemistry and Chemical Engineering 7 (12): 1142-8.

[16] Qi, L., Yin, Z., Zhang, S., Ouyang, Q., Li, C., and Chen, Y. 2014. "The Increased Interface Charge Transfer in Dye-sensitized Solar Cells based on Well-ordered $\mathrm{TiO}_{2}$ Nanotube Arrays with Different Lengths.” J. Mater. Res. 29 (6): 745-75.

[17] Sun, J., Gao, L., and Zhang, Q. H. 2003. "Synthesizing and Comparing the Photocatalytic Properties of High Surface Area Rutile and Anatase Titania Nanoparticles." J. Am. Ceram. Soc. 86 (10): 1677-82.

[18] Mayabadi, A. H., Waman, V. S., Kamble, M. M., Ghosh, S. S., Gabhale, B. B., and Rondiya, S. R. et al. 2014. "Evolution of Structural and Optical Properties of Rutile $\mathrm{TiO}_{2}$ Thin Films Synthesized at Room Temperature by Chemical Bath Deposition Method." Journal of Physics and Chemistry of Solids 75 (2): 182-7. 